\title{
Economic Status Inequality Is a Predictor for Screening and Health Utilization
}

\author{
Yousef Veisani' , Ali Delpisheh², Salman Khazaei ${ }^{3, *}$ \\ ${ }^{1}$ Psychosocial Injuries Research Center, Ilam University of Medical Sciences, Ilam, Iran \\ ${ }^{2}$ Department of Clinical Epidemiology, Ilam University of Medical Sciences, Ilam, Iran \\ ${ }^{3}$ Department of Epidemiology, School of Public Health, Hamadan University of Medical Sciences, Hamadan, Iran
}

\section{To the Editor}

The direct link between socioeconomic status (SES) and health outcomes is well known, especially for non-communicable diseases such as diabetes, hypertension, and mental disorders. ${ }^{1-3)}$

We read the article titled "Disparity in Health Screening and Health Utilization according to Economic Status" by Kim et al., ${ }^{4)}$ published in the July 2017 issue of the Korean Journal of Family Medicine. Kim et al. ${ }^{4)}$ showed a correlation between lower economic status and lower rate of health screening attendance and post-screening outpatient visits in people aged over 40 years. This is an interesting article with important implications in the context of creating action plans for screening and prevention programs to deal with health issues such as cardiovascular disease. Nevertheless, we would like to make two observations about the study.

The three main data sources for inequality analysis are surveillance systems and population- and institution-based sources. The authors of the article obtained their data from a cohort database, which is similar to a surveillance systems database. An advantage of surveillance systems data is that it can provide data on an individual disease or from a selected location. This feature is useful for reducing over- and underreporting of data. In contrast, it has some disadvantages, including its relatively fewer equity stratifies, which may affect the allocation of resources to some groups. Furthermore, the data obtained is not always adequately representative. Combining small-area identifiers in a single reign could help address this problem. ${ }^{5)}$

In their article, Kim et al. ${ }^{4)}$ used the simple measures (odds ratio) to estimate the inequality between different SES categories with regard to health screening attendance rates and postscreening outpatient visits. An important limitation of simple measures is that they do not take all the subgroups (the five economic status categories) into consideration. Moreover, only two groups have been compared, and the methodology ignores the remaining subgroups. Therefore, simple measures with a pairwise scale cannot indicate the full extent of inequality trends in the five categories. On the other hand, complex measures such as concentration index present a single expression of inequality calculated across all subgroups and do not have this limitation. ${ }^{6}$

In conclusion, this study shows interesting results. However, when interpreting these results, it is essential to consider the concerns about data sourcing and inequality measures described above.

\section{CONFLICT OF INTEREST}

No potential conflict of interest relevant to this article was reported.

\section{REFERENCES}

1. Veisani Y, Moradi G, Delpisheh A. Effects of socio-economic status inequality on health outcomes. Arch Iran Med 2017;20: 329.

2. Veisani Y, Delpisheh A. Decomposing of socioeconomic inequality in mental health: a cross-sectional study into femaleheaded households. J Res Health Sci 2015;15:218-22.

3. Fateh M, Emamian MH, Asgari F, Alami A, Fotouhi A. Socioeconomic inequality in hypertension in Iran. J Hypertens 
2014;32:1782-8.

4. Kim MJ, Lee H, Kim EH, Cho MH, Shin DW, Yun JM, et al. Disparity in health screening and health utilization according to economic status. Korean J Fam Med 2017;38:220-5.

5. Li Y, Wu Q, Xu L, Legge D, Hao Y, Gao L, et al. Factors affecting cata- strophic health expenditure and impoverishment from medical expenses in China: policy implications of universal health insurance. Bull World Health Organ 2012;90:664-71.

6. Pearcy JN, Keppel KG. A summary measure of health disparity. Public Health Rep 2002;117:273-80. 